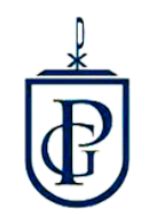

Veritas Et Scientia

Vol. $7, N^{\circ} 1,877-889$

Enero - Junio del 2018

ISSN $2307-5139$

\title{
DETERMINACIÓN DEL TIPO DE SUELO Y POTENCIAL DE COLAPSO DEL SUELO DEL PUESTO DE SALUD INTIORKO DISTRITO DE CIUDAD NUEVA, DEPARTAMENTO DE TACNA-2017.
}

DETERMINATION OF THE SOIL AND POTENTIAL TYPE OF SOIL COLLAPSE OF THE INTIORKO HEALTH PLACE DISTRICT OF CIUDAD NUEVA, DEPARTMENT OF TACNA-2017

Alfonso Oswaldo Flores Mello ${ }^{1}$

Presentado: $18 / 02 / 2018$

Aceptado: 05/05/2018

Publicado online: $11 / 06 / 2018$

\section{RESUMEN}

Se investigó el tipo de suelo y potencial de colapso del suelo del Puesto de Salud Intiorko, distrito de Ciudad Nueva, departamento de Tacna. Se desarrolló la investigación aplicada, debido que se utilizó conocimiento pre existente en investigaciones y normas técnicas, analizando información existente cercana a la zona de estudio, desarrollando ensayos estándares para la identificación de suelos colapsables, así como ensayos de colapso en laboratorio e in situ mediante la prueba de carga con colapso de la estructura del suelo para determinar el potencial de colapso. Se identificó que las propiedades físicas, mecánicas y químicas contribuyen a la estructura colapsable del suelo, esto debido a la baja densidad natural, límite líquido, alta relación de vacíos y sales solubles totales; así mismo, identificó que a la presión de $1.00 \mathrm{~kg} / \mathrm{cm} 2$ no se genera problemas de colapso, mientras que con 2.00 $\mathrm{kg} / \mathrm{cm} 2$ el problema es moderado; igualmente se identificó que el asentamiento por colapso corresponde al $90 \%$ del asentamiento inmediato producto de la prueba de carga in situ con colapso. El potencial de colapso del suelo del Puesto de Salud Intiorko, distrito de Ciudad Nueva, departamento de Tacna, para capacidad de carga admisible de $1.00 \mathrm{~kg} / \mathrm{cm} 2$ es moderado, debido que las propiedades físicas, mecánicas y químicas contribuye en el potencial de colapso y a esta presión la distorsión angular no produce agrietamiento en muros.

Palabras clave: suelo, colapso, capacidad de carga admisible

\footnotetext{
${ }^{1}$ Maestría en ingeniería civil con mención en geotecnia
} 


\begin{abstract}
The type of soil and soil collapse potential of the Intiorko Health Post, district of Ciudad Nueva, department of Tacna, was investigated. Applied research was developed, due to the use of preexisting knowledge in research and technical standards, analyzing existing information close to the study area, developing standard tests for the identification of collapsible soils, as well as laboratory and in situ collapse tests. The load test with collapse of the soil structure to determine the potential for collapse. It was identified that the physical, mechanical and chemical properties contribute to the collapsible structure of the soil, this due to the low natural density, liquid limit, high ratio of voids and total soluble salts; Likewise, he identified that at the pressure of $1.00 \mathrm{~kg} / \mathrm{cm} 2$ collapse problems are not generated, while with $2.00 \mathrm{~kg} / \mathrm{cm} 2$ the problem is moderate; It was also identified that the settlement by collapse corresponds to $90 \%$ of the immediate settlement product of the in situ load test with collapse. The soil collapse potential of the Intiorko Health Station, in Ciudad Nueva, Tacna department, for a permissible load capacity of 1.00 $\mathrm{kg} / \mathrm{cm} 2$ is moderate, because the physical, mechanical and chemical properties contribute to the potential for collapse and this pressure the angular distortion does not cause cracking in walls.
\end{abstract}

Keywords: soil, collapse, allowable load capacity

\title{
INTRODUCCIÓN
}

En la presente investigación se analiza el potencial de colapso del suelo del Puesto de Salud Intiorko, distrito de Ciudad Nueva, departamento de Tacna, con base a ensayos in-situ y comparándolos con ensayos realizados en laboratorio, para comprender el comportamiento frente al incremento del contenido de humedad por agentes externos. Esto debido, a su baja densidad seca y contenido de humedad natural, así como su elevada relación de vacíos y variable contenido de sales solubles totales, lo cual hace propenso que la estructura del suelo colapse debido a un aumento del contenido de humedad por agentes externos y se presenten asentamientos diferenciales, lo que conlleva fisuras en los elementos estructurales de la edificación. Estas propiedades fueron obtenidas en base a los resultados de los ensayos estándares, colapso en laboratorio y colapso in situ, los cuales rebelan contenido de material cementante, el cual proporciona una rigidez y resistencia considerable, y un potencial de colapso moderado, recomendando presiones admisibles menores a $1.00 \mathrm{~kg} / \mathrm{cm}^{2}$. El distrito de Ciudad Nueva de la provincia y departamento de Tacna, en el cual se ubica el puesto de salud Intiorko, en la Asociación de vivienda 28 de Agosto, fue materia de análisis en la tesis "Estudio de suelos para cimentaciones en edificaciones del cono norte de la ciudad de Tacna" (Silva - Berrios 1998), la cual con base a ensayo de corte directo y colapso, obtuvo capacidades de carga y asentamiento máximos para este distrito, clasificando el área en estudio como Zona E: Suelo muy suelto y determinó la capacidad portante del suelo entre $0.30 \mathrm{~kg} / \mathrm{cm}^{2}$ a $0.50 \mathrm{~kg} / \mathrm{cm}^{2}$. Respecto a la sismicidad del departamento de Tacna y de la zona sur del Perú y norte de Chile, se sabe que debido a que el último sismo de intensidad mayor a IX o X ocurrió el 13 de Agosto de 1868, es decir a la fecha, han pasado 150 años sin la ocurrencia de sismos similares; y considerando que los especialistas indican que terremotos de esta intensidad tienen periodos de recurrencia cada 150 a 270 años, la región sur del Perú y Norte de Chile son muy propensas 
a la ocurrencia de sismos de la citada intensidad. Los estudios geotécnicos en el Perú utilizan técnicas muy importantes como son la exploración de campo, los ensayos de laboratorio y los ensayos in situ, con base en la norma técnica peruana E.050 Suelos y cimentaciones aprobado mediante decreto supremo n.ㅇ 011-2006-VIVIENDA el 8 de mayo de 2006 .

En los suelos áridos y semiáridos generalmente se encuentran suelos colapsables. Estos suelos son secos, es decir el contenido de humedad son bajos; sin embargo, son resistentes en su estado natural y proporcionan buenas condiciones para las cimentaciones, debido que contienen material cementante, que unen los sólidos. Aunque, al incrementar el contenido de humedad, el material cementante que unía las partículas sólidas del suelo, son disueltas por el agua, produciendo un reacomodo de las partículas y con ello un asentamiento conforme se rompa las uniones cementadas, generando un asentamiento inesperado y localizado en las zonas que, por alguna razón se humedece. Sin embargo, en estado seco, estos suelos son estables y tienen buen comportamiento mecánico. Para evitar este tipo de asentamientos, las cimentaciones deben ser proyectadas identificando el suelo colapsable, evaluando el asentamiento por colapso y emplear las medidas de mitigación apropiadas cuando fuera necesario, según concluyeron Clemence y Finbarr, 1981.

Braja M. Das, define estos suelos colapsables, también llamados metaestables, como suelos no saturados que experimentan un gran cambio de volumen al incrementar el contenido de humedad y/o al saturarse y en algunas veces a un incremento de una carga adicional.

Jenning y Knight (1975) fueron unos de primeros en realizar investigaciones sobre suelos colapsables, ellos recomendaron un procedimiento para determinar el potencial de colapso de un suelo, el cual consiste en obtener una muestra de suelo inalterado con su contenido de humedad natural en un anillo de consolidación, muestra de aproximadamente $5 \mathrm{~cm}$. de diámetro y $2 \mathrm{~cm}$. de altura, aplicar cargas escalonadas hasta la presión de $2 \mathrm{~kg} / \mathrm{cm}^{2}$, luego se inunda la muestra de suelo hasta saturarla y se deja reposar. Determinándose las relaciones de vacíos antes y después de la saturación, $\mathbf{e}_{1}$ y $\mathbf{e}_{2}$, calculando con ello el potencial de colapso $\mathbf{C p}$, respecto a la relación de vacíos inicial $\mathrm{e}_{0}$.

En las cimentaciones superficiales, la capacidad de carga admisible es la menor de entre la capacidad de carga que ocasiona la capacidad de carga por corte dividida entre el factor de seguridad y el asentamiento admisible que la estructura puede soportar. Lo cual se detalla en la norma peruana E.050 Suelos y cimentaciones, artículo 3.5.

\section{METODOLOGÍA}

Investigación experimental, centrada básicamente en el comportamiento esfuerzo vs deformación, por medio de la ejecución de ensayos en el Laboratorio de Mecánica de Suelos, Concretos y Pavimentos de la Universidad Privada de Tacna y in situ, para determinar el potencial de colapso del suelo bajo análisis, del Puesto de Salud Intiorko, distrito de Ciudad Nueva, departamento de Tacna. Para la presente investigación, se tiene como antecedentes tres calicatas, de las cuales se desarrollaron los ensayos estándares y especiales, así como la prueba in situ de carga en placa sin colapso en la misma zona de estudio, en ese sentido adicionalmente se plantea realizar una calicata adicional con ensayos de laboratorio estándares y especiales adicionales como colapso unidimensional; así como la prueba in situ de carga en placa con colapso. Así mismo, para el análisis se consideró parámetros físicos, químicos y mecánicos de investigaciones anteriores cercanas a la zona de estudio, descritos en los antecedentes. Para identificar los parámetros físicos del suelo bajo estudio, se desarrollaron ensayos estándares con la medición de Densidad in situ (norma NTP 339.143 ASTM D 1556), Contenido de humedad (norma NTP 339.127 ASTM D 2216), Granulometría (norma NTP 339.128 ASTM D422), Límites de Atterberg (norma NTP 339.129 ASTM D 4318)

\section{OBJETIVOS}

- Obtener las propiedades físicas, mecánicas, químicas y potencial de colapso en laboratorio del suelo del Puesto de Salud Intiorko, distrito de Ciudad Nueva, departamento de Tacna. 
- Aplicar el ensayo de colapso insitu para determinar el potencial de colapso del suelo del Puesto de Salud Intiorko, distrito de Ciudad Nueva, departamento de Tacna.

\section{RESULTADOS}

Se puede resumir en base a las investigaciones anteriores, que el suelo de la zona de estudio está clasificado como Arena Limosa (SM), límite líquido menor a 30\%, no presenta límite plástico, contenido de humedad menor al 5\%, densidad relativa menor al 40\%, gravedad específica de los sólidos alrededor de $2.60 \mathrm{gr} / \mathrm{cm}^{3}$. En ensayos de corte directo en laboratorio con muestras remoldeadas, se determinó que el ángulo de fricción varía desde 22.00 a 22.89 o y la cohesión $\mathrm{c}=0.00$ a $0.277 \mathrm{~kg} / \mathrm{cm}^{2}$. Igualmente, utilizando el equipo triaxial y ensayo no consolidado no drenado, se determinó el ángulo de fricción de 260 y cohesión $\mathrm{c}=0.00 \mathrm{~kg} / \mathrm{cm}^{2}$. De otro lado, realizando pruebas in situ, por medio del ensayo de carga en placa, se determinó el ángulo de fricción de 30.00 y cohesión $\mathrm{c}=0.00 \mathrm{~kg} / \mathrm{cm}^{2}$. Adicionalmente a ello, en el distrito de Ciudad Nueva, dependiendo de la densidad y contenido de sales solubles totales, el ángulo de fricción puede varias de 22.12 a a 36.60 y la cohesión desde 0.275 a $0.335 \mathrm{~kg} / \mathrm{cm}^{2}$. Respecto al módulo de elasticidad, se determinaron por medio de muestras remoldeadas valores entre 98 a 275 $\mathrm{kg} / \mathrm{cm}^{2}$ mientras que en pruebas in situ $440 \mathrm{~kg} / \mathrm{cm}^{2}$, mediante el ensayo de carga en placa, y de 139 a $992 \mathrm{~kg} / \mathrm{cm}^{2}$, utilizando el ensayo MASW, según la tesis "Caracterización dinámica de los suelos en la ciudad de Tacna". De la revisión a ensayos históricos del contenido de sales solubles totales en el distrito de Ciudad Nueva, se advierte que el límite máximo obtenido en la Universidad Privada de Tacna, es 28 370 ppm. Según investigaciones anteriores, con muestras remoldeadas, el potencial de colapso del suelo del distrito de ciudad Nueva puede llegar hasta el $7.70 \%$. Adicionalmente a ello, entre las densidades secas de 1.30 a $1.50 \mathrm{gr} / \mathrm{cm}^{3}$ y contenido menores de $30000 \mathrm{ppm}$ de sales solubles totales, el potencial de colapso es inferior al $5.00 \%$.

Tabla 01: Resumen de ensayos estándares.

\begin{tabular}{|c|c|c|c|c|c|c|c|c|c|c|c|c|c|}
\hline$\frac{\mathbb{\pi}}{\stackrel{0}{\pi}}$ & 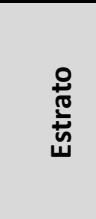 & $\begin{array}{l}\text { 을 } \\
\frac{\pi}{0} \\
\frac{0}{0} \\
5 \\
\frac{5}{2} \\
\frac{0}{2}\end{array}$ & 气̆ & 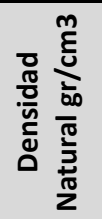 & 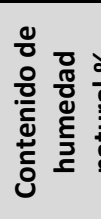 & 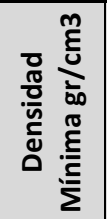 & 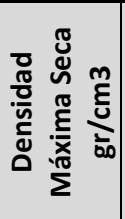 & ذ̊ & 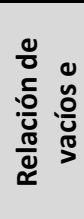 & 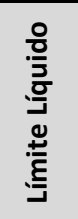 & 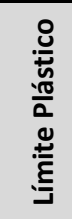 & 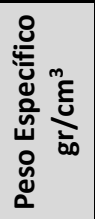 & 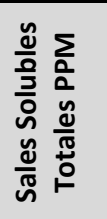 \\
\hline \multirow{2}{*}{ C-01 } & E - 02 & 2.00 & SM & 1.41 & 2.01 & 1.37 & 1.69 & $18 \%$ & $88 \%$ & $21 \%$ & NP & 2.62 & -- \\
\hline & $E-02$ & 3.00 & SM & 1.43 & 1.89 & 1.37 & 1.71 & $22 \%$ & $88 \%$ & $22 \%$ & NP & 2.63 & 2910 \\
\hline
\end{tabular}

\begin{tabular}{|l|l|l|l|l|l|l|l|l|l|l|l|l|l|}
\hline \multirow{2}{*}{ C-01 } & E - 01 & 0.30 & SM & 1.41 & 2.08 & 1.38 & 1.66 & $11 \%$ & $88 \%$ & $22 \%$ & NP & 2.59 & 14040 \\
\cline { 2 - 14 } & E - 02 & 0.90 & SM & 1.52 & 1.76 & 1.38 & 1.70 & $49 \%$ & $75 \%$ & $26 \%$ & NP & 2.61 & 23040 \\
\hline \multirow{3}{*}{ C-02 } & E - 01 & 0.70 & SM & 1.40 & 4.90 & 1.38 & - & - & $88 \%$ & $23 \%$ & NP & 2.51 & 14680 \\
\cline { 2 - 14 } & E - 02 & 0.80 & SM & 1.43 & 1.25 & 1.38 & 1.69 & $18 \%$ & $88 \%$ & $28 \%$ & NP & 2.66 & 28640 \\
\cline { 2 - 13 } & E - 03 & 1.10 & SM & 1.53 & 1.48 & 1.38 & 1.68 & $55 \%$ & $75 \%$ & $26 \%$ & NP & 2.65 & 32800 \\
\hline \multirow{2}{*}{ C-03 } & E - 01 & 0.30 & SM & 1.39 & 3.20 & 1.23 & 1.68 & $43 \%$ & $89 \%$ & $24 \%$ & NP & 2.56 & 15120 \\
\cline { 2 - 13 } & E - 02 & 0.90 & SM & 1.49 & 3.21 & 1.23 & 1.69 & $63 \%$ & $72 \%$ & $27 \%$ & NP & 2.50 & 27560 \\
\hline
\end{tabular}

Fuente: Ensayos estándares y Sales Solubles Totales, Apéndices A-01, 02, 03, 04, 05, 06, 07 y 08.

Con base a la propuesta de metodología, se determinaron las características físicas del suelo, la primera calicata es la realizada en la presente investigación y las tres siguientes fueron las ejecutadas en la Tesis "Determinación de los parámetros de la resistencia al esfuerzo cortante del Puesto de Salud Intiorko de la Asociación de vivienda 28 de agosto - Ciudad Nueva - Tacna" ubicadas en la misma edificación y realizadas en el año 2008: Como se puede apreciar del cuadro resumen anterior, el suelo típico de la zona de estudio, se presenta a 1.00 metro de profundidad, el cual contiene grumos compuestos de arena, limo y sales de aproximadamente de 1" a 2" de diámetro, suelo que según la clasificación SUCS 
es Arena Limosa SM no plástica. Así mismo, al nivel de cimentación la densidad natural seca va desde $1.41 \mathrm{gr} / \mathrm{cm}^{3}$ a $1.53 \mathrm{gr} / \mathrm{cm}^{3}$, límite líquido entre $21 \%$ y $27 \%$ y contenido de humedad de $1.76 \%$ a $3.21 \%$ , el cual es típico de suelos areno limosos colapsables.

\section{ENSAYO DE COLAPSO EN LABORATORIO}

\section{Método de doble edómetro}

Aplicando el método descrito con dos muestras, la primera en su estado natural y la segunda con humedecimiento (saturación) inicial se obtuvo la siguiente gráfica, de la cual se aprecia que ambas curvas son casi paralelas entre sí.

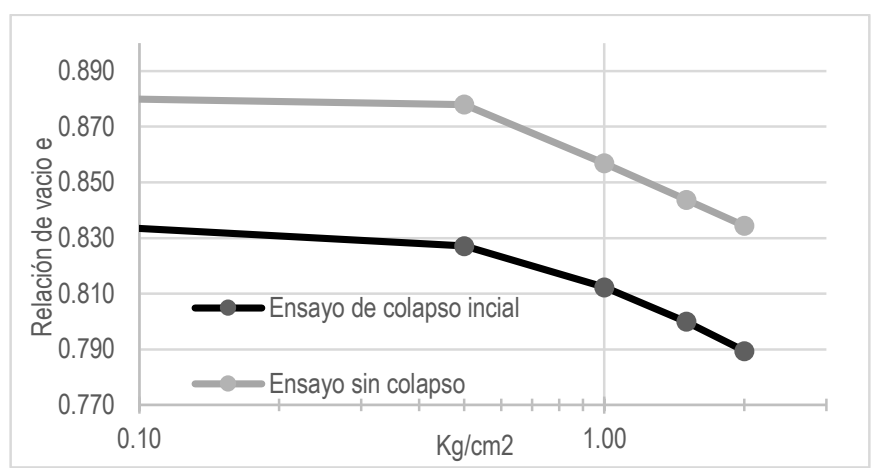

Gráfico n. 1: Ensayo de colapso - doble edómetro

Tabla 1: Ensayo de colapso inicial

\begin{tabular}{|c|c|c|c|}
\hline \multicolumn{2}{|c|}{$\begin{array}{c}\text { Ensayo con colapso } \\
\text { inicial }\end{array}$} & \multicolumn{2}{c|}{ Ensayo sin colapso } \\
\hline $\begin{array}{l}\text { Carga } \\
\mathbf{k g} / \mathbf{c m}^{2}\end{array}$ & $\begin{array}{l}\text { Relación de } \\
\text { vacíos e }\end{array}$ & $\begin{array}{l}\text { Carga } \\
\mathbf{k g} / \mathbf{c m}^{2}\end{array}$ & $\begin{array}{l}\text { Relación de } \\
\text { vacíos e }\end{array}$ \\
\hline 0.01 & 0.883 & 0.01 & 0.883 \\
\hline 0.01 & 0.842 & 0.50 & 0.878 \\
\hline 0.50 & 0.827 & 1.00 & 0.857 \\
\hline 1.00 & 0.812 & 1.50 & 0.844 \\
\hline 1.50 & 0.800 & 2.00 & 0.834 \\
\hline 2.00 & 0.789 & & \\
\hline
\end{tabular}

\section{Método de simple edómetro}

Los ensayos especiales que se realizaron fueron: colapso unidireccional en $1.00 \mathrm{~kg} / \mathrm{cm}^{2}$ y $2.00 \mathrm{~kg} / \mathrm{cm}^{2}$. Al respecto, el primero de ello, se realizó con muestra remoldeada a densidad natural de $1.45 \mathrm{gr} / \mathrm{cm}^{3}$, iniciando el ensayo a $0.50 \mathrm{~kg} / \mathrm{cm}^{2}$ e incrementando a $1.00 \mathrm{~kg} / \mathrm{cm}^{2}$, luego de mantenerse el asentamiento, se humedeció la muestra y posteriormente se aumentó la carga a $1.50 \mathrm{~kg} / \mathrm{cm}^{2}$ y $2.00 \mathrm{~kg} / \mathrm{cm}^{2}$, obteniendo la siguientes gráfica relación de vacíos $(\mathrm{e})-$ carga $\mathrm{kg} / \mathrm{cm}^{2}$, según se aprecia en gráfico 2 :

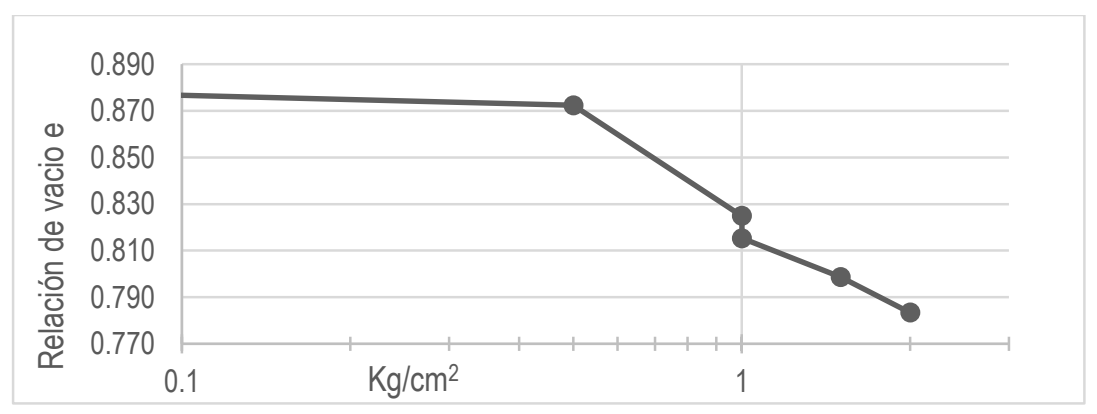

Gráfico 2: Ensayo de colapso a $1 \mathrm{~kg} / \mathrm{cm}^{2}$. 


\begin{tabular}{|c|r|}
\hline Carga $\mathbf{~ k g} / \mathbf{c m}^{2}$ & $\begin{array}{l}\text { Relación de } \\
\text { vacios e }\end{array}$ \\
\hline 0.01 & 0.883 \\
\hline 0.50 & 0.872 \\
\hline 1.00 & 0.825 \\
\hline 1.00 - Saturación de muestra & 0.815 \\
\hline 1.50 & 0.799 \\
\hline 2.00 & 0.783 \\
\hline
\end{tabular}

Así mismo, se realizó el mismo ensayo, pero el humedecimiento (colapso) del suelo se aplicó en la carga de $2 \mathrm{~kg} / \mathrm{cm}^{2}$, obteniendo gráfico 3 :

Gráfico 3: Ensayo de colapso a $2 \mathrm{~kg} / \mathrm{cm}^{2}$.

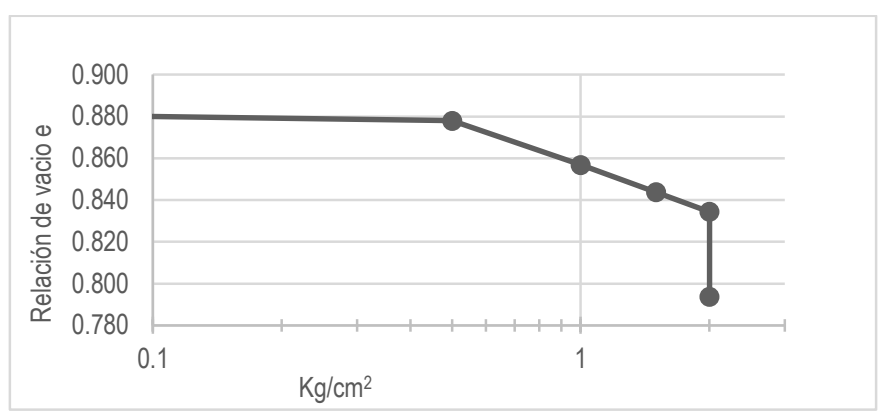

\begin{tabular}{|c|r|}
\hline Carga $\mathbf{~ k g} / \mathrm{cm}^{2}$ & Relación de vacíos e \\
\hline 0.01 & \\
\hline 0.50 & 0.883 \\
\hline 1.00 & 0.878 \\
\hline 1.50 & 0.857 \\
\hline 2.00 & 0.844 \\
\hline 2.00 - Saturación de muestra & 0.834 \\
\hline
\end{tabular}

Fuente: Ensayo de colapso.

\section{ENSAYO DE COLAPSO IN SITU}

Para el desarrollo del colapso in situ se optó por realizar el ensayo con carga en placa in situ de 0.30 metros de diámetro, a una profundidad de 2.00 metros, correspondiente a la zona activa de la cimentación de la edificación, y saturación del suelo al esfuerzo cercano a $1 \mathrm{~kg} / \mathrm{cm}^{2}$ según se aprecia en tabla 2:

Tabla 02: Resumen de resultados del ensayo de colapso in situ.

\begin{tabular}{|c|c|c|c|c|c|c|c|c|c|}
\hline \multicolumn{2}{|c|}{ Dial-01 } & \multicolumn{2}{c|}{ Dial-02 } & \multicolumn{2}{c|}{ Dial-03 } & \multicolumn{2}{c|}{ Promedio } & \multicolumn{2}{c|}{$\begin{array}{c}\text { Promedio } \\
\text { corregido }\end{array}$} \\
\hline $\mathrm{Kg} / \mathbf{c m}^{2}$ & $\mathbf{m m}$ & $\mathbf{K g} / \mathbf{c m}^{2}$ & $\mathbf{m m}$ & $\mathbf{K g} / \mathbf{c m}^{2}$ & $\mathbf{m m}$ & $\mathbf{K g} / \mathbf{c m}^{2}$ & $\mathbf{m m}$ & $\mathbf{K g} / \mathbf{c m}^{2}$ & $\mathbf{m m}$ \\
\hline 0.01 & 0.01 & 0.01 & 0.01 & 0.01 & 0.01 & 0.01 & 0.01 & 0.01 & 0.01 \\
\hline 0.43 & 0.23 & 0.43 & 0.35 & 0.43 & 0.40 & 0.43 & 0.33 & 0.43 & 0.13 \\
\hline 0.72 & 0.33 & 0.72 & 0.43 & 0.72 & 0.42 & 0.72 & 0.39 & 0.72 & 0.19 \\
\hline 0.94 & 0.41 & 0.94 & 0.51 & 0.94 & 0.45 & 0.94 & 0.45 & 0.94 & 0.25 \\
\hline 0.94 & 0.53 & 0.94 & 0.89 & 0.94 & 0.63 & 0.94 & 0.68 & 0.94 & 0.48 \\
\hline 1.95 & 0.56 & 1.95 & 1.04 & 1.95 & 0.77 & 1.95 & 0.79 & 1.95 & 0.59 \\
\hline
\end{tabular}


Como se aprecia en el gráfico 4, la curva presión vs deformación de las mediciones durante el ensayo in situ, no se advierte la fracción inicial lineal, motivo por el cual se corrigió los datos obtenidos, según se aprecia:

Gráfico 4: Asentamiento promedio del ensayo de colapso in situ.

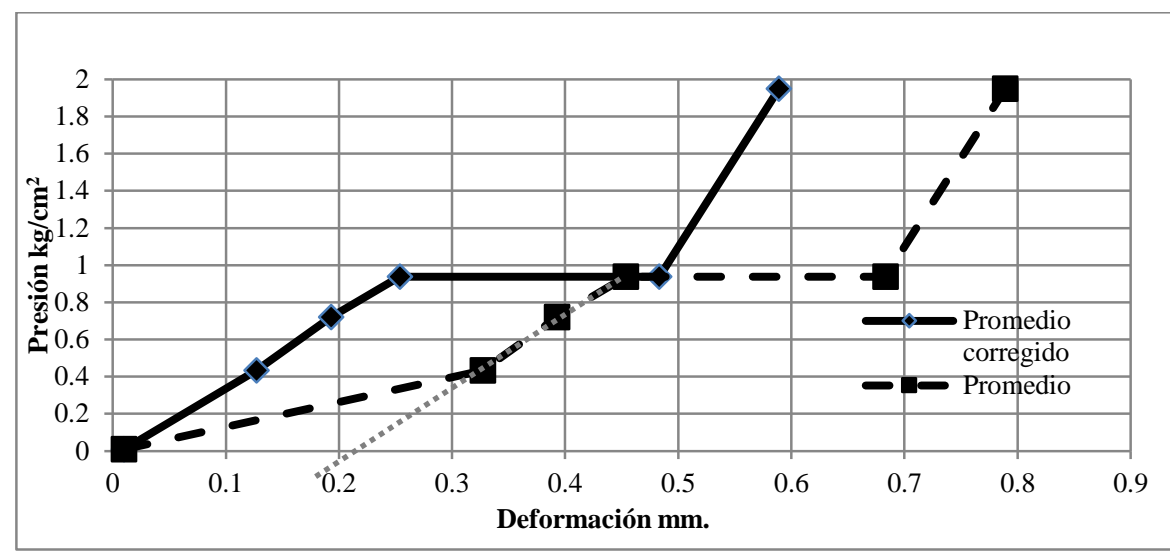

\section{VALIDACIÓN DE LA METODOLOGÍA PARA DETERMINAR EL POTENCIAL DE COLAPSO PARÁMETROS FÍSICOS}

Al nivel de cimentación la densidad natural seca se encuentra, desde $1.41 \mathrm{gr} / \mathrm{cm}^{3}$ a $1.53 \mathrm{gr} / \mathrm{cm}^{3}$, límite líquido entre $21 \%$ y $27 \%$ y contenido de humedad de $1.76 \%$ a $3.21 \%$, parámetros físicos que son típico de suelos areno limosos colapsables; en ese sentido, considerando el gráfico propuesto por Holtz y Hilf y definido por la norma peruana E 0.50 Suelos y Cimentaciones, el presente suelo es colapsable, según se aprecia en gráfico 5 :

Gráfico 5: Relación entre suelos colapsables y no colapsables.

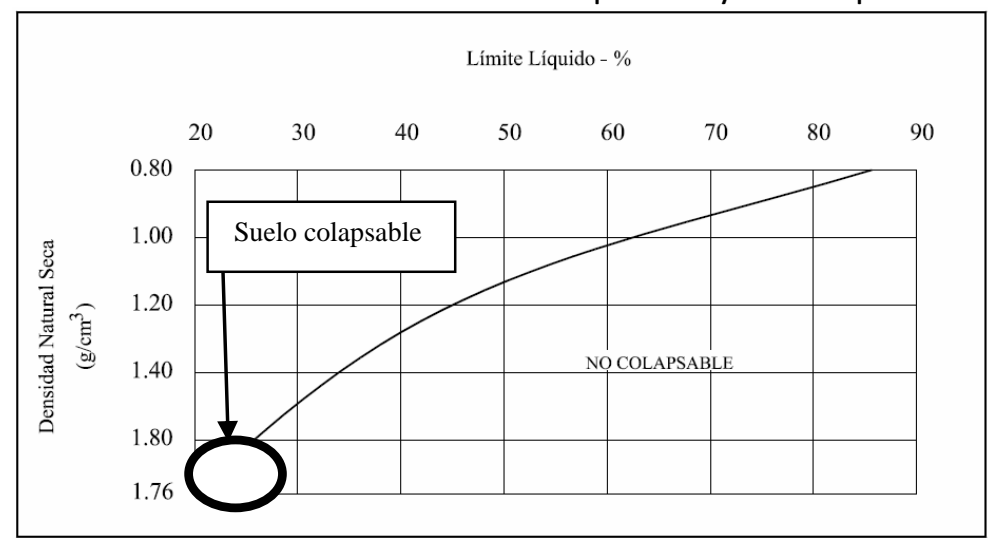

Fuente: Norma técnica E 0.50 Suelos y cimentaciones, figura n. ${ }^{\circ}$ 6.1.2

Igualmente, las densidades mínima (Apéndice A-04) y máximas (Apéndice A-05) son entre $1.23 \mathrm{gr} / \mathrm{cm}^{3}$ y $1.71 \mathrm{gr} / \mathrm{cm}^{3}$ respectivamente, obteniendo densidades relativas entre el $18 \%$ al $63 \%$ y relación de vacíos entre 0.75 y 0.89 , siendo estos últimos elevados. En ese sentido, utilizando las fórmulas propuestas por Denisov (Factor K), Gibbs (factor R) y código soviético (factor L), se determinó cualitativamente que el suelo es colapsable. , según se detalla en la tabla 3 : 
Tabla 3: Resumen de factores de suelos colapsables.

\begin{tabular}{|c|c|c|c|c|c|c|c|c|c|c|c|}
\hline \multirow[b]{2}{*}{ Descripción } & \multirow[b]{2}{*}{ Nomenclatura } & \multirow[b]{2}{*}{ Uni. } & \multicolumn{2}{|c|}{ CALICATA 01 } & \multicolumn{2}{|c|}{ CALICATA 01 } & \multicolumn{3}{|c|}{ CALICATA 02 } & \multicolumn{2}{|c|}{ CALICATA 03 } \\
\hline & & & \begin{tabular}{|l|} 
E- $02-$ \\
$2,00 \mathrm{~m}$
\end{tabular} & \begin{tabular}{|l|} 
E- 02 - \\
$3,00 \mathrm{~m}$
\end{tabular} & E- 01 & E- 02 & E-01 & E- 02 & E- 03 & E- 01 & E- 02 \\
\hline Volumen de Sólidos & $V s=\frac{W s}{\gamma_{s}}$ & $\mathrm{cc}$ & 38,17 & 38,02 & 38,61 & 38,31 & 39,89 & 37,64 & 37,72 & 39,12 & 40,06 \\
\hline Volumen de Agua & $V w=\frac{W w}{\left(\gamma_{o}\right)}$ & $\mathrm{cc}$ & 2,00 & 2,00 & 2,08 & 1,76 & 4,90 & 1,25 & 1,48 & 3,20 & 3,21 \\
\hline Volumen de Vacíos & $V v=V m-V s$ & $\mathrm{cc}$ & 33,66 & 33,31 & 33,79 & 28,63 & 35,04 & 33,17 & 28,61 & 35,12 & 29,20 \\
\hline Relación de Vacíos & $e=\frac{V v}{V s}$ & -- & 0,88 & 0,88 & 0,88 & 0,75 & 0,88 & 0,88 & 0,76 & 0,90 & 0,73 \\
\hline Grado de Saturación & $G s \%=\frac{V w}{V v} \times 100$ & -- & $5,94 \%$ & $6,00 \%$ & $6,16 \%$ & $6,15 \%$ & $13,98 \%$ & $3,77 \%$ & $5,17 \%$ & $9,11 \%$ & $10,99 \%$ \\
\hline Límite líquido & $\mathrm{LL}$ & -- & 0,21 & 0,22 & 0,22 & 0,26 & 0,23 & 0,28 & 0,26 & 0,24 & 0,27 \\
\hline Peso de agua al Límite líquido & WwLL & $\mathrm{gr}$ & 21,00 & 22,00 & 22,00 & 26,00 & 23,00 & 28,00 & 26,00 & 24,00 & 27,00 \\
\hline $\begin{array}{l}\text { Volumen de agua al Lìmite } \\
\text { líquido }\end{array}$ & VwLL & $\mathrm{cc}$ & 21,00 & 22,00 & 22,00 & 26,00 & 23,00 & 28,00 & 26,00 & 24,00 & 27,00 \\
\hline $\begin{array}{l}\text { Relación de vacìos al Límite } \\
\text { líquido }\end{array}$ & eLL & & 0,55 & 0,58 & 0,57 & 0,68 & 0,58 & 0,74 & 0,69 & 0,61 & 0,67 \\
\hline $\begin{array}{l}\text { Factor } \mathrm{K} \\
\mathrm{K}=0.50 \text { a } 0.75 \text { muy colaps able. } \\
\mathrm{K}=1.00 \text { no colapsable. } \\
\mathrm{K}=1.50 \text { a } 2.00 \text { no colapsa. }\end{array}$ & eLUe & & 0,624 & 0,661 & 0,651 & 0,908 & 0,656 & 0,844 & 0,909 & 0,683 & 0,925 \\
\hline $\begin{array}{l}\text { Contenido de humedad en } \\
\text { saturación }\end{array}$ & $\mathrm{W} \%$ (sat) & & 0,337 & 0,333 & 0,338 & 0,286 & 0,350 & 0,332 & 0,286 & 0,351 & 0,292 \\
\hline $\begin{array}{l}\text { Factor } \mathrm{R} \\
\text { Si es mayor a 1, el suelo colapsa }\end{array}$ & W\% (sat) / LL & & 1,603 & 1,514 & 1,536 & 1,101 & 1,523 & 1,185 & 1,100 & 1,463 & 1,082 \\
\hline $\begin{array}{l}\text { Factor L } \\
\text { Para un grado de saturación } \\
\text { natural menor que } 60 \%, \text { si L >- } \\
0.10 \text {, se trata de un suelo } \\
\text { colapsable. }\end{array}$ & $L=\frac{e 0-e L}{1+e 0}$ & & 0,176 & 0,159 & 0,163 & 0,039 & 0,161 & 0,073 & 0,039 & 0,150 & 0,032 \\
\hline
\end{tabular}

Otro factor muy importante y que contribuye a que este suelo es colapsable, es el alto y variable contenido de sales de la zona de estudios alrededor de 27800 ppm, los cuales cementan las partículas sólidas del suelo, proporcionando resistencia adicional al esfuerzo cortante del suelo, que luego de humedecido, este pierde su resistencia y con ello ocasiona asentamientos diferenciales

\section{ENSAYO DE COLAPSO EN LABORATORIO Método de simple edómetro}

Tal como se detalló, el colapso unidireccional se realizó a $1.00 \mathrm{~kg} / \mathrm{cm}^{2}$ y $2.00 \mathrm{~kg} / \mathrm{cm}^{2}$. En el primer caso se determinó el potencial de colapso $0.51 \%$, es decir, según lo propuesto por Clemence y Finbarr y definido en la norma peruana E0.50 Suelos y Cimentaciones, la severidad del problema es catalogado como "Ningún Problema" al ser menor al 1\%; por lo que, el presente suelo con esta magnitud de esfuerzo aplicado es no colapsable, según se aprecia en la gráfica

Gráfico 5: Ensayo de colapso a $1 \mathrm{~kg} / \mathrm{cm}^{2}$.

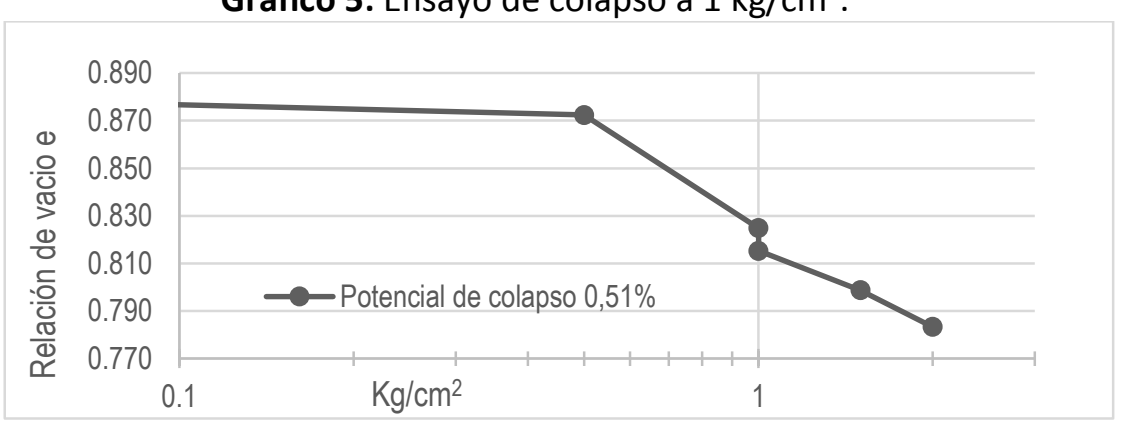

Fuente: Ensayo de colapso. 
En el segundo caso, al humedecer (colapso) el suelo durante la carga de $2.00 \mathrm{~kg} / \mathrm{cm}^{2}$, se determinó el potencial de colapso de $2.16 \%$, que según lo propuesto por Clemence y Finbarr y definido en la norma peruana E0.50 Suelos y Cimentaciones, corresponde a "Problema Moderado"; por lo que para la presente carga de esfuerzo, el suelo es colapsable, según se aprecia en el gráfico 7.

Gráfico 6: Ensayo de colapso a $2 \mathrm{~kg} / \mathrm{cm}^{2}$

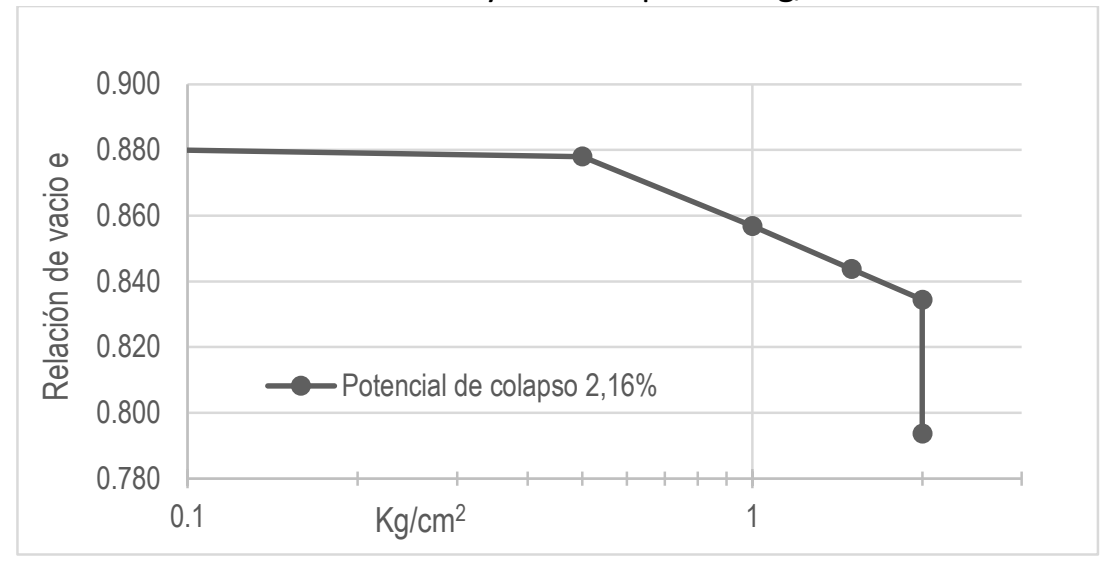

Fuente: Ensayo de colapso.

Ahora bien, superponiendo ambos ensayos, se aprecia que la curva de relación de vacíos vs esfuerzo, no son paralelas entre las cargas de $0.50 \mathrm{~kg} / \mathrm{cm}^{2}$ a $1.00 \mathrm{~kg} / \mathrm{cm}^{2}$; sin embargo, entre las cargas de 1.00 a $2.00 \mathrm{~kg} / \mathrm{cm}^{2}$, si son paralelas, según se aprecia en el gráfico 8 .

Gráfico 7 : Superposición de los ensayos de colapso a $1 \mathrm{~kg} / \mathrm{cm}^{2}$ y $2 \mathrm{~kg} / \mathrm{cm}^{2}$.

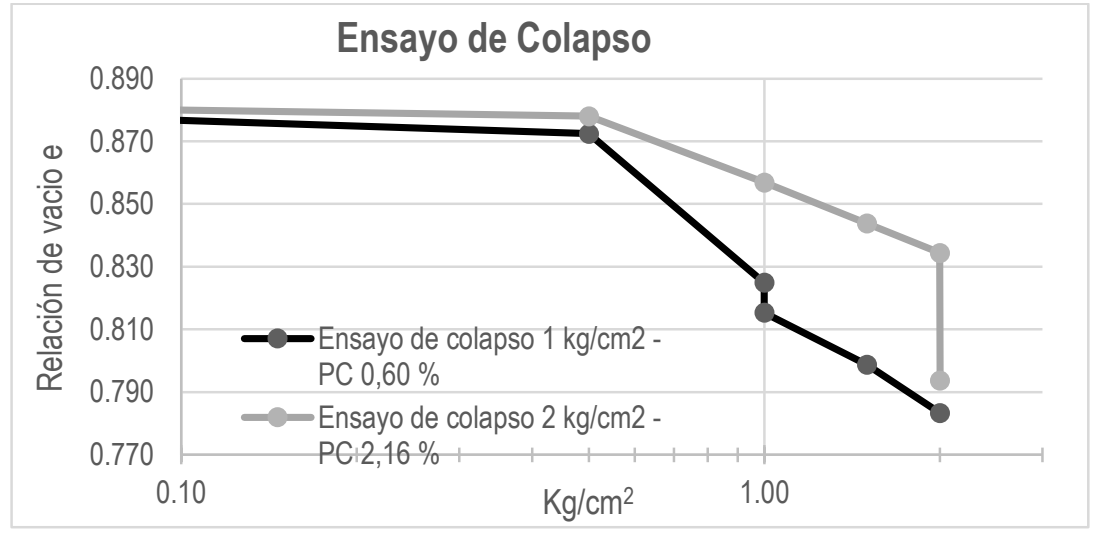

Fuente: Ensayo de colapso.

Adicionalmente a ello, las relaciones de vacíos finales son similares para ambos ensayos.

\section{Método de doble edómetro}

Respecto a este método, se determinó la gráfica relación de vacíos - presión para dos muestras, la primera en estado natural y la segunda con colapso inicial, según se aprecia en la gráfica 9. 
Gráfico 8: Ensayo de colapso - doble edómetro.

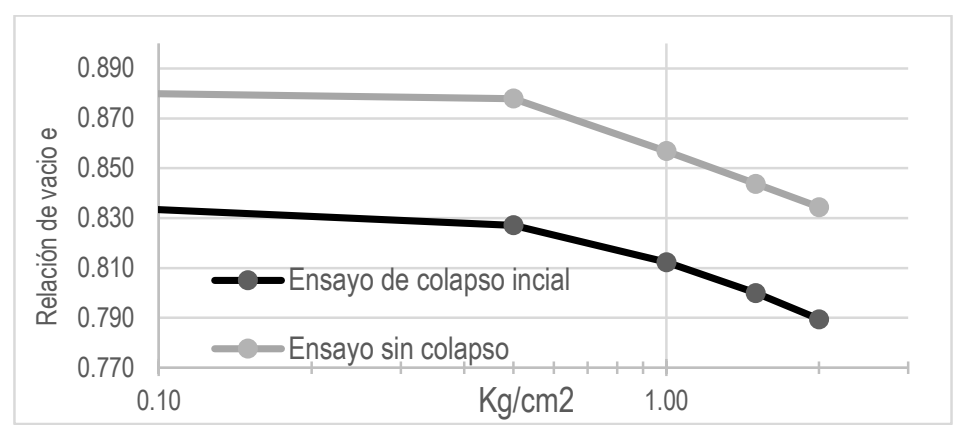

Fuente: Ensayo de colapso del doble edómetro.

Con base a estas curvas, para una zapata cuadrada de $1.20 \mathrm{~m}$. de lado con capacidad admisible de $1 \mathrm{~kg} / \mathrm{cm}^{2}$ y $2 \mathrm{~kg} / \mathrm{cm}^{2}$, profundidad de desplante $2 \mathrm{~m}$. y asumiendo una profundidad de análisis de 2.40 $\mathrm{m}$., se procedió calcular los asentamientos elástico, $\mathrm{Sc}_{(1)}$, y por colapso, $\mathrm{Sc}_{(2)}$.

Determinando que el rango del asentamiento por colapso es de $68 \%$ a $89 \%$ del asentamiento elástico; además que, el asentamiento por colapso se incrementa conforme aumenta la presión sobre el suelo, es decir cuanto mayor capacidad admisible se considere mayor será el asentamiento por colapso, según se resume en la tabla 4:

Tabla 4: Asentamiento elástico y de colapso con esfuerzos de $1 \mathrm{~kg} / \mathrm{cm}^{2}$

y $2 \mathrm{~kg} / \mathrm{cm}^{2}$.

\begin{tabular}{|l|r|r|r|}
\hline $\begin{array}{c}\text { Capacidad } \\
\text { Admisible }\end{array}$ & Sc (1) $\mathbf{m}$. & Sc (2) $\mathbf{~ m . ~}$ & \multicolumn{1}{|c|}{$\begin{array}{c}\text { Relación } \\
\text { Sc(2)/Sc(1) }\end{array}$} \\
\hline $1 \mathrm{~kg} / \mathrm{cm}^{2}$ & 0.022 & 0.020 & $89 \%$ \\
\hline $2 \mathrm{~kg} / \mathrm{cm}^{2}$ & 0.036 & 0.025 & $68 \%$ \\
\hline
\end{tabular}

Fuente: Elaboración propia, Apéndice A-13 y A-14.

Es de precisar que, este asentamiento es obtenido asumiendo que únicamente existe asentamiento vertical y el estrato afectado por el colapso es de $2.40 \mathrm{~m}$.

\section{ENSAYO DE COLAPSO IN SITU}

De la curva presión vs deformación del ensayo in situ, se determinó el asentamiento elástico de $0.25 \mathrm{~mm}$. para la carga de $0.94 \mathrm{~kg} / \mathrm{cm}^{2}$, y luego de la aplicación del colapso se incrementó en $0.23 \mathrm{~mm}$., es decir el asentamiento por colapso es cercano al $90 \%$ del asentamiento elástico, según se aprecia:

Gráfico 9: Asentamiento promedio del ensayo de colapso in situ.

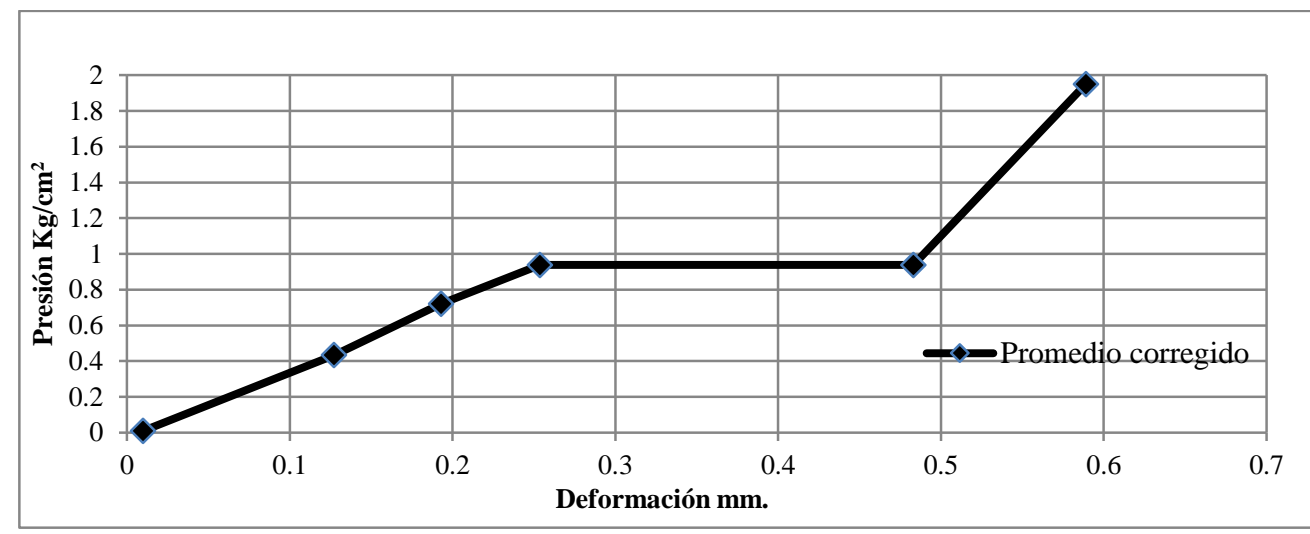

Fuente: Ensayo de colapso in situ. 
Adicionalmente a ello, en la gráfica se advierte un cambio de pendiente entre el suelo antes y después del colapso y/o saturación del suelo. Ahora bien, al superponer estas curvas con la prueba de carga in situ con colapso de la presente investigación, se advierte que el último ensayo (CP-Colapso) tiene una mayor pendiente en la zona previa al colapso que los dos ensayos anteriores (CP-01 y CP-02), lo que equivale a un mayor módulo de elasticidad y parámetros de resistencia al esfuerzo cortante, esto probablemente obedece a la mayor profundidad de análisis, cambio de contenido de sales y/o densidad in situ, según se aprecia en la gráfica 11.

Gráfico 10: Ensayo de carga en placa y colapso

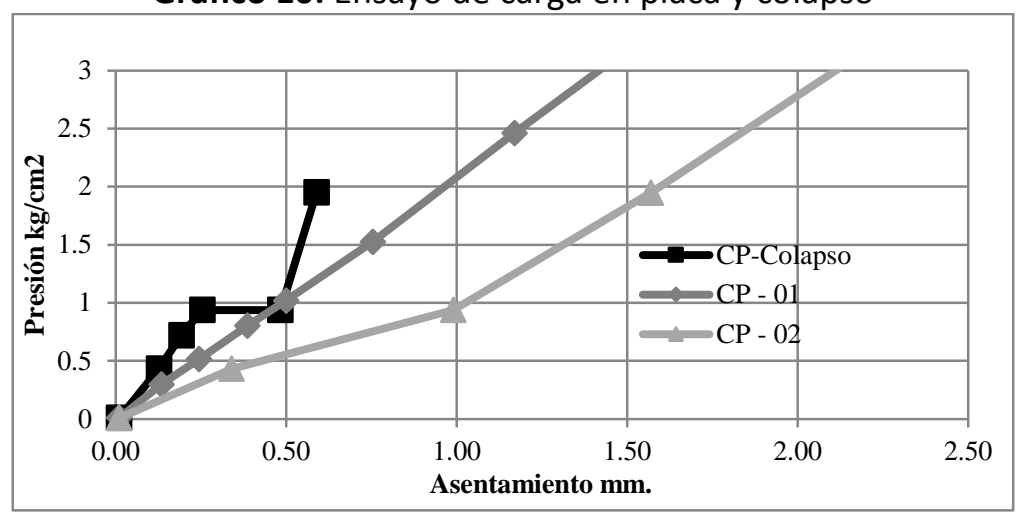

Por lo que, se aprecia variaciones de las características físicas, mecánicas y químicas de la zona de estudio.

\section{DISCUSIÓN}

Considerando las características físicas del suelo bajo análisis, se determinó cualitativamente que el suelo es colapsable, debido básicamente a su baja densidad natural y por tanto elevada relación de vacíos, reducido límite líquido, así como su variable contenido de sales solubles totales, el cual es directamente proporcional al potencial de colapso. Así mismo, los ensayos de colapso unidimensional determinaron que el suelo presenta problema moderado cuando en la presión de $2.00 \mathrm{~kg} / \mathrm{cm}^{2} \mathrm{se}$ produce el colapso; mientras que no presenta problemas cuando el colapso se produce en $1.00 \mathrm{~kg} / \mathrm{cm}^{2}$. Respecto al asentamiento producido por el colapso, tanto en análisis teóricos (método del doble edómetro) y ensayos in situ (prueba de carga con colaps), se advierte que el asentamiento correspondiente al colapso, se encuentra entre el 69 y $90 \%$ del asentamiento inmediato.Adicionalmente a ello, Terzaghi y Peck en 1967 propusieron la expresión para determinar los asentamientos inmediatos y/o elásticos de cimentaciones en función al ensayo de carga directa. En ese sentido, con base al ensayo de carga en placa se determinó el asentamiento inmediato en función de la prueba de carga directa, en zapatas cuadradas típicas de 1,20 metros de lado, con capacidades admisibles de $1.00,1.50$ y 2.00 $\mathrm{kg} / \mathrm{cm}^{2}$. Adicionalmente a ello, considerando que la norma técnica peruana E.050 Suelos y Cimentaciones, define que el asentamiento inmediato diferencial es de $75 \%$ del asentamiento inmediato y/o elástico, y que el asentamiento debido al colapso del suelo es de alrededor del $90 \%$ del asentamiento inmediato, se determinó que la distorsión (1/695) de la zapata con capacidad de carga admisible de 1.00 $\mathrm{kg} / \mathrm{cm}^{2}$ sin colapso, es menor al límite propuesto por la norma técnica peruana para edificios en los que no se permiten grietas (1/300). Mientras que, considerando el colapso del suelo, la distorsión se ve reducida a 1/316, límite en el cual se debe esperar grietas, sin daño estructural. La distorsión de las zapatas con capacidad de carga admisible de 1.50 y $2.00 \mathrm{~kg} / \mathrm{cm}^{2} \sin$ y con colapso, son menores a los límites propuestos por la norma, al sobrepasar $1 / 300$ que corresponde al límite en que se debe esperar las primeras grietas en paredes y al estar muy cercano a 1/150 que representa el límite en el que se debe esperar daño estructural en edificios convencionales.

En ese sentido, se advierte que guarda proporcionalidad con lo observado en laboratorio, al 
realizarse el humedecimiento a un esfuerzo de $1.00 \mathrm{~kg} / \mathrm{cm}^{2}$, en el cual se obtuvo que el suelo o colapsa, frente al esfuerzo de $2.00 \mathrm{~kg} / \mathrm{cm}^{2}$, en el cual el colapso es moderado. Si consideramos que sobre la zapata se aplicará una carga de $1.00 \mathrm{~kg} / \mathrm{cm}^{2}$, el asentamiento por colapso no causará distorsiones mayores a los máximos permitidos; sin embargo con forme aumentamos la carga sobre la zapata, es decir $2.00 \mathrm{~kg} / \mathrm{cm}^{2}$, el asentamiento por colapso causaría distorsiones cercanos los límites permitidos por la norma. Motivo por el cual, el colapso del suelo del suelo del Puesto de Salud Intiorko, distrito de Ciudad Nueva, departamento de Tacna es leve, debido que las cargas soportadas por las zapatas son menores de 1.00 $\mathrm{kg} / \mathrm{cm}^{2}$. Por lo que, no se recomienda capacidades admisibles mayores a $1.00 \mathrm{~kg} / \mathrm{cm}^{2}$; asimismo para este tipo de problema especial del suelo, se recomienda el uso de materiales resistentes al ataque químico y vigas de cimentación en ambas direcciones y rigidizar en lo posible la menor dirección, con el fin de contrarrestar el deterioro prematuro de la subestructura y disminuir los asentamientos diferenciales (que pueden ser causados por el asentamiento por colapso del suelo) en la cimentación en las posibilidades de la economía, según el esquema propuesto por Clemense y Finbarr.

Para el diseño de cimentaciones de todo proyecto de mediana envergadura, se debe realizar estudios en detalle de mecánica de suelos, los cuales deben tener como mínimo los ensayos estándares como densidad in situ seca y límite líquido y utilizar la gráfica propuesta por Holtz y Hilf y definido por la norma técnica peruana E 0.50 Suelos y Cimentaciones, ya que esta es aplicable a este tipo de suelo areno limoso, para determinar la necesidad de realizar mayor análisis del posible colapso del suelo. No se recomienda la gráfica propuesta por Holtz y Hilf y definido por la norma técnica peruana E.050 Suelos y Cimentaciones para suelos gravosos y/o con bolonería, debido a que alta densidad puede ocultar un posible potencial de colapso, tal como ocurre en algunas zonas del distrito de Gregorio Albarracín Lanchipa, en la ciudad de Tacna. Utilizar como información preliminar el potencial de colapso determinado con diferentes densidades y contenidos de sales solubles totales, de la investigación "Influencia del contenido de sales solubles totales en el potencial de colapso del limo arenoso en la institución educativa Cesar Augusto Cohaila Tamayo № 42250 del distrito de Ciudad Nueva - provincia de Tacna". Es necesario utilizar como información preliminar los parámetros de resistencia al esfuerzo cortante del suelo determinado con diferentes densidades y contenidos de sales solubles totales, de la investigación "Influencia del contenido de sales solubles totales en los parámetros de resistencia al esfuerzo cortante del limo arenoso en la institución educativa. Cesar Augusto Cohaila Tamayo № 42250 del distrito de Ciudad Nueva - provincia de Tacna". Se recomienda el uso del ensayo de carga en placa, debido a que nos permite conocer el comportamiento esfuerzo - deformación del suelo en estado natural, pudiendo determinar el esfuerzo para el cual las deformaciones son proporcionales a los esfuerzos y asentamiento debido al colapso de la estructura del suelo por incremento del contenido de humedad. Asimismo se recomienda el uso de vigas de cimentación o cimentaciones conectadas para contrarrestar los posibles asentamientos producidos por la presencia de humedad en edificaciones y redistribuir los esfuerzos.

\section{REFERENCIAS BIBLIOGRÁFICAS}

Amer Ali, State of the Art Review of Collapsible Soil, (2000).

Alva Hurtado, Diseño de cimentaciones superficiales. Instituto de la construcción y gerencia.

Alvares Y Piccardo, Estudio de arenas colapsables de la región industrial de Matanzas. (1988).

Badillo Y Rodriguez, Mecánica de suelos tomo I y Tomo II. (1985) Editorial Limusa.

Braja M. Das, Cimentaciones. (2001) Editorial THOMSON

Braja M. Das, Cimentaciones. (2006) Editorial THOMSON

Braja M. Das, Principios de ingeniería de cimentaciones. (2006) Editorial THOMSON.

Caterina, Maria, Identification and behavior of collapsible soils. (2012).

Crespo V., Mecánica de suelos y cimentaciones. (2004) Editorial Limusa.

Condori Quispe, Betty María, Tesis: Investigación del conglomerado especial en la Ciudad de Tacna, 2012.

Day, Robert W., Foundation engineering handbook: Design and construction with the 2006 international 
building code. (2006) Editorial McGraw-Hill Companies.

Donald P. Coduto, Fundation design principles and practices. (2001) Editorial Prentice Hall

Fernandez Sixto, Erasmo Alejandro, Tesis: Investigación del conglomerado colapsable de la Cano-Vitor Arequipa, 1998.

Flores Mello, Alfonso Oswaldo, Tesis: "Determinación de los parámetros de la resistencia al esfuerzo cortante del Puesto de Salud Intiorko de la Asociación de vivienda 28 de Agosto - Ciudad Nueva Tacna", 2010.

Flores Kuong, Emmanuel Hugo Y Rospigliosi Rengifo, Raul German, Tesis: "Influencia del contenido de sales solubles totales en los parámetros de resistencia al esfuerzo cortante del limo arenoso en la institución educativa. Cesar Augusto Cohaila Tamayo № 42250 del distrito de Ciudad Nueva provincia de Tacna", 2015.

Instituto Nacional De Defensa Civil, Universidad Nacional Jorge Basadre Goohmann Y Programa De Las Naciones Unidas Para El Desarrollo - "Estudio Mapas de Peligro para la Ciudad de Tacna y Locumba" 1998

Instituto Nacional De Defensa Civil Y Programa De Las Naciones Unidas Para El Desarrollo - Ciudades Sostenibles, 2002.

Lambe, Ingeniería de cimentaciones. Editorial Limusa.

Norma Técnica Peruana E.050 Suelos y cimentaciones, 2006.

Peck, Hanson Y Thornburn, Ingeniería de cimentaciones. (2002) Editorial Limusa.

Redolfi, Emilio, Suelos colapsables, (2007).

Silva a. Y Berrios M., "Estudio de suelos para cimentaciones en edificaciones del cono norte de la Ciudad de Tacna" Tesis. Tacna-Perú. 